\title{
Complications of Long-Term Opioid Therapy for Management of Chronic Pain: the Paradox of Opioid-Induced Hyperalgesia
}

\author{
D. Eric Brush
}

Published online: 15 September 2012

(C) American College of Medical Toxicology 2012

\begin{abstract}
While opioids remain a valid and effective analgesic strategy for patients suffering from a wide variety of painful conditions, they are not a panacea. Increasingly, physicians must balance patient expectations of adequate pain control with known limitations of opioid pharmaceuticals including adverse effects, tolerance, addiction, withdrawal, and drug diversion. Further complicating the issue over the last decade is a growing body of evidence suggesting chronic opioid use may unexpectedly worsen the perception of pain in some individuals. This syndrome, termed opioid-induced hyperalgesia $(\mathrm{OIH})$, fundamentally changes our understanding of opioid pharmacodynamics and may influence our approach to management of chronic pain. This manuscript describes the concept $\mathrm{OIH}$ and provides an overview of basic science and clinical research to date attempting to characterize this syndrome, as well as ascertain its clinical relevance. The potential existence of $\mathrm{OIH}$ in humans is framed within the context of our current understanding of opioids and our prescribing patterns so that physicians may begin to incorporate these ideas into their philosophy of pain management as further information develops. Animal studies reliably validate OIH in controlled models. Rigorous research protocols in humans are lacking, and we cannot yet confidently conclude that OIH manifests in clinically significant ways. However, clinicians should consider the possibility of $\mathrm{OIH}$ when evaluating outcomes of patients on chronic opioid therapy.
\end{abstract}

Keywords Opioids $\cdot$ Hyperalgesia $\cdot$ Opioid-induced hyperalgesia $\cdot$ Chronic pain

\footnotetext{
D. E. Brush $(\bowtie)$

Department of Emergency Medicine, Division of Toxicology, UMass Medical Center,

Worcester, MA, USA

e-mail: brushd@umassmemorial.org
}

\section{Current Opioid Paradigm}

Physicians regularly prescribe opioids for control of moderate to severe pain in the acute care setting. This includes administration in emergency, outpatient, and inpatient environments for patients suffering from a variety of acute and chronic painful conditions. Our current paradigm for opioid utilization suggests they provide effective analgesia for short-term use in most patients. Unfortunately, the risk of adverse effects or addiction is not rare and may be difficult to accurately quantify [1]. Regular prescription of opioids also occurs in the long-term care setting for patients with cancer-related pain offering effective pain relief and limited concern for addiction. However, long-term use of opioids for treatment of certain chronic conditions such as back pain, fibromyalgia, or neuropathic pain syndromes sparks more debate regarding therapeutic efficacy, adverse effects, and potential for misuse, abuse, and diversion of prescription opioids. Concerns regarding opioid use for patients with chronic non-cancer pain may relate to a subset of these patients who require significant dose escalation over time, visit multiple providers seeking opioid prescriptions, and report insufficient pain relief despite high dose therapy. These scenarios generate several concerns including the possibility that chronic opioid administration may be an ineffective strategy for long-term analgesia. Emerging evidence suggests that in some patients chronic opioid exposure may actually worsen the perception of pain.

\section{Opioid Interactions with Nociceptive Pathways}

Nociception describes complex pathways that enable the perception of pain triggered by noxious stimuli. The experience of pain is adaptive, facilitating the learning process whereby we take action to shield ourselves from harm. 
Conversely, pain becomes maladaptive when experienced as severe and ongoing beyond the point of directing behavior modification. Endogenous opioid receptor ligands, for example enkaphalin, provide intrinsic anti-nociceptive balance. In the normal state, pro- and anti-nociceptive pathways work in harmony facilitating an adaptive experience of pain without undue suffering. Exogenous opioid administration aims to tip the balance toward an antinociceptive state wherein analgesia supersedes pain. Acutely, this strategy provides effective control of pain. However, evidence presented below demonstrates that the complex biochemical and neuroanatomical pathways of nociception may adapt to our interventions producing unintended maladaptive consequences.

In a simplified overview of the normal state, exogenous opioids bind to inhibitory G-protein-coupled receptors producing three key downstream effects [2]. First, through inhibition of adenylate cyclase (AC), intracellular concentrations of cyclic AMP (cAMP) decrease. Reduced phosphorylation and activation of multiple proteins, enzymes, and ion channels diminishes excitatory activity in nociceptive pathways. Opioid binding affects impulse conduction via two other mechanisms: blockade of calcium channels, and facilitation of potassium ion influx. Reduced intracellular calcium prevents exocytosis of vesicles containing excitatory amino acids and neuropeptides such as glutamate, substance P (SP), and calcitonin gene-related peptide (CGRP). Hyperpolarization of the post-synaptic neuron via potassium ion influx has a concordant effect of limiting neurotransmission across the synapse.

Opioids exert their primary analgesic effects at both spinal and supraspinal levels [3]. For example, inhibited phosphorylation of the post-synaptic $N$-methyl-D-aspartate receptor (NMDA-r) in the dorsal horn of the spinal cord decreases its sensitivity to glutamate binding. Therefore, transmission of a pain signal from the periphery is muted upon synapsing in the spinal cord. Similarly, diminished synaptic exocytosis of glutamate, SP, and CGRP in response to afferent pain signals reduces transmission beyond the spinal level. Centrally, opioids bind in the periaqueductal gray matter (PAG) and attenuate activity in descending fibers to the rostral ventromedial medulla (RVM)[4]. The "off-cells" in the RVM are normally quiescent in response to tonic inhibition from the PAG. However, when opioids reduce this tonic inhibition, "off-cells" become active and send downward inhibitory influence to the spinal cord creating a blockade for afferent nociceptive signals bound for the CNS. In this way opioid binding at spinal and supraspinal levels mitigates our awareness of noxious stimuli.

Analgesia from opioid therapy can be limited by tolerance, a familiar expected pharmacologic outcome in response to prolonged receptor agonism from an exogenous ligand. With respect to opioid receptors this results in blunting of the usual anti-nociceptive effect of opioids necessitating dose escalation to yield the desired effect. Associated cellular changes include opioid receptor phosphorylation, internalization, and sequestration leading to downregulation of available receptors [5]. In response to cessation of opioid therapy, or de-escalation of dosing, patients can experience withdrawal and may report increased pain sensitivity in this context.

\section{Syndrome of Opioid-Induced Hyperalgesia}

Opioid-induced hyperalgesia $(\mathrm{OIH})$, a novel unanticipated outcome, may also develop following prolonged opioid therapy. This phenomenon is characterized by a heightened perception of pain related to the use of opioids in the absence of disease progression or opioid withdrawal. Stated otherwise opioids may directly facilitate pro-nociceptive pathways such that patients are overly affected by noxious stimuli compared with opioid naïve patients. Receptor downregulation, a model used to explain tolerance, fails to adequately explain how this could occur.

Research efforts struggle to develop consensus definition of $\mathrm{OIH}$ and incorporate this into experimental protocols designed to identify this effect. Experimental observation of pain attenuation in both animals and humans is often measured with respect to pain tolerance. The validity of operationally defining OIH by a reduction in baseline pain tolerance induced by opioid exposure remains unclear. Another variable, the threshold at which pain is perceived, would be challenging in animal models, and may be too subjective in humans to demonstrate consistent results. Therefore, a reduction in baseline pain tolerance in response to opioid exposure has emerged as the defining measurement of $\mathrm{OIH}$ in most studies.

\section{Clinical Observations Supporting OIH}

Reports as early as 1870 allude to the occurrence of $\mathrm{OIH}$ in morphine-addicted patients [6]. Physicians with experience prescribing long-term opioid therapy may embrace intuitive assumptions regarding the existence of $\mathrm{OIH}$ in the absence of validated surveillance tools. Perhaps the patient on methadone reporting severe pain with ordinary venipuncture could suffer from OIH. A patient on high doses of oxycodone for chronic back pain may rate the pain intensity from a mild ankle strain on the numeric pain scale as a 10 (scale $0-10$ ), whereas we would typically expect a lower rating in the average patient. Without clearly defined criteria this diagnosis remains a suspicion under these circumstances and could also be explained as progression of disease states, opioid tolerance, withdrawal, or $\mathrm{OIH}$. If identified 
correctly, pharmacologic tolerance or progression of underlying disease may respond appropriately to increased opioid dosing, whereas manifestations of OIH could, in theory, intensify with higher dosing [7].

\section{Animal Data Supporting Existence of OIH}

Numerous animal models aim to illustrate $\mathrm{OIH}$ utilizing pain tolerance to a wide variety of noxious stimuli including heat, electric, and chemical irritants. Perhaps the simplest and most instructive is the rat model of hind paw withdrawal. The Hargreave apparatus is a small plastic cube that houses the rat during experimental protocol. The floor of the cube is rapidly heated using an infrared lamp. Time zero marks the start of the heating process, and the time of hind paw withdrawal from the floor in seconds indicates the animal's pain tolerance. Stated differently, the animal's tolerance for pain is measured as paw withdrawal latency. A typical research design using this technique establishes two groups of rats: group A has a surgically implanted osmotic pump delivering saline while group B receives a pump delivering a constant rate of an opioid [8]. Both groups are tested for baseline paw withdrawal latency on day 0 prior to pump implantation. Figure 1, a representative graph, demonstrates the observed effect verified by multiple studies utilizing a variety of opioids. The increase in paw withdrawal latency after the initial onset of opioid infusion is not surprising, nor is the return to baseline latency that we ascribe to tolerance. The decreased latency that develops after a period of time on opioid therapy, however, indicates a diminished pain tolerance consistent with

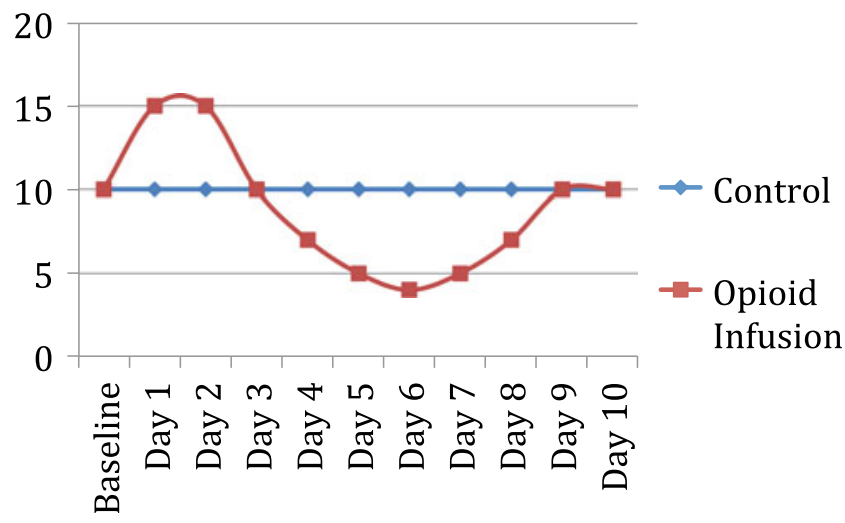

Fig. 1 Paw withdrawal latency demonstrating OIH. Data are representative of studies conducted in rats using a thermal stimulus to the hind paw. The $y$-axis indicates seconds of exposure prior to paw withdrawal. Baseline measurements for control and opioid-exposed groups verify no difference in withdrawal latency. Opioid exposure on day 1 increases the withdrawal latency and indicates analgesia. Return to baseline on day 3 suggests tolerance, and subsequent decreased latency below pre-opioid exposure measurements illustrates the occurrence of OIH. Removal of opioid exposure on day 7 results in a return to baseline
OIH. Removal of the opioid infusion pump eventually restores the withdrawal latency to baseline. In a 2006 review, Angst and Clark compiled a comprehensive table of similar experiments identifying $\mathrm{OIH}$ in animal models [9].

\section{Basic Science Research Examining Mechanisms of OIH}

In vivo studies reveal that contrary to our current understanding of downstream activity produced acutely by opioid receptor binding, chronic opioid exposure produces a distortion of biochemical signaling. Following acute exposure to morphine, cultured cells exhibit inhibition of AC activity [10]. Paradoxically, chronic exposure enhances the activity of AC. Following termination of opioid exposure, AC activity returns to baseline. This "superactivation" of AC effectively reverses the typical effects of opioids outlined above. Similar studies demonstrate that chronic opioid exposure paradoxically activates a variety of protein kinases (PK) such as PKC [11]. Subsequent upregulation of NMDA receptors and downregulation of opioid receptors, facilitated by PKC activation, provides further insight into the mechanisms driving OIH. NMDA receptors appear to play a pivotal role as shown in animal models of OIH $[11,12]$. In these studies, the presence of a potent NMDA receptor antagonist, MK801, prevents the occurrence of $\mathrm{OIH}$. Colocalization of NMDA and opioid receptors, along with a propensity for chronic opioid exposure to facilitate NMDA receptor-specific pathways such as PKC activation, leads to the concept of "second messenger switching [13]." Thus, although opioids lack affinity for NMDA receptors, binding at the opioid receptor may functionally produce effects expected from its co-localized neighbor. A myriad of biochemical derangements resulting from chronic opioid exposure have been identified and help further demystify the pathophysiology underlying OIH (Table 1).

\section{Human Data Supporting OIH}

Studying OIH in humans involves much higher complexity and more careful interpretation than with animal models. First, there is no defined clinical syndrome describing OIH in humans, and no widely accepted operational definition of OIH driving experimental consistency. Therefore, outcome measures between studies vary, limiting comparison. Second, most studies enroll patients already taking opioids for a variety of medical conditions. This non-random exposure to opioids creates multiple confounding issues. Studies must identify and control for tolerance to opioids, withdrawal states, and personality profiles associated with opioid addiction. Research design must also consider progression of underlying disease states and pre-existing or developing hyperalgesia that may be 
Table 1 Compartmentalized analysis of mechanisms related to the occurrence of $\mathrm{OIH}$

\begin{tabular}{|c|c|c|}
\hline Compartment & Target of interest & Effect of chronic opioids \\
\hline Peripheral & $\begin{array}{l}\text { TRPV1, non-specific ion channel transduces stim- } \\
\text { uli into afferent pain signal }\end{array}$ & $\begin{array}{l}\uparrow \text { transcription of TRPV1 in dorsal root ganglia with translocation to } \\
\text { periphery [23] }\end{array}$ \\
\hline \multirow[t]{12}{*}{ Spinal } & $\begin{array}{l}\text { NMDA receptors co-localized with opioid recep- } \\
\text { tors in dorsal horn }\end{array}$ & $\begin{array}{l}\text { Blockade with MK801 prevents OIH. Evidence of "second messenger } \\
\text { switching" } \\
\uparrow \text { release of EAA and } \uparrow \text { sensitivity to EAA [11] }\end{array}$ \\
\hline & \multirow{4}{*}{$\begin{array}{l}\text { PKC, not normally activated by inhibitory G- } \\
\text { protein receptor activation }\end{array}$} & Paradoxical activation of $\mathrm{PKC}$ \\
\hline & & Opioid-r downregulation \\
\hline & & NMDA-r upregulation \\
\hline & & $\begin{array}{l}\uparrow \text { transcription factor such as CREB leads to } \uparrow \text { expression of CGRP and SP } \\
\text { [11] }\end{array}$ \\
\hline & \multirow[t]{5}{*}{ Adenylate cyclase } & Paradoxical activation \\
\hline & & $\uparrow \mathrm{cAMP}$ \\
\hline & & $\uparrow E A A$ receptor activation \\
\hline & & $\uparrow$ Calcium entry and vesicle exocytosis \\
\hline & & $\downarrow$ Potassium entry, loss of hyperpolarization $[10]$ \\
\hline & \multirow{2}{*}{$\begin{array}{l}\text { Dynorphin, analgesic in normal state, binds to } \\
\text { Kappa receptor }\end{array}$} & $\uparrow$ spinal dynorphin \\
\hline & & $\uparrow$ release of SP and CGRP via NMDA-r dependent mechanism $[12,24]$ \\
\hline \multirow[t]{2}{*}{ Central } & \multirow[t]{2}{*}{$\mathrm{CCK}$} & $\uparrow \mathrm{CCK}$ expression occurs in response to opioids [25] \\
\hline & & $\begin{array}{l}\text { Blocks opioid-related "off-cell" disinhibition in the RVM attenuating } \\
\text { downward projecting spinal inhibition [26] }\end{array}$ \\
\hline
\end{tabular}

This table identifies various neurotransmitters, neuropeptides, receptors, and other biochemical targets that undergo modifications in response to opioid exposure. These disruptions in normal physiology are postulated to underlie the experimentally produced phenomenon of $\mathrm{OIH}$

TRPV1 transient receptor potential vanilloid-1, NMDA N-methyl-D-aspartate, EAA excitatory amino acid, PKC protein kinase C, CREB cAMPresponse element binding protein, $C G R P$ calcitonin gene-related peptide, $S P$ substance $\mathrm{P}, C C K$ cholecystokinin, $R V M$ rostral ventromedial medulla

associated with some pain syndromes. Further, the morphine3-glucoronomide metabolite of morphine can induce hyperalgesia and allodynia (perception of pain in response to nonnoxious stimuli) and highlights the need for consideration of agent-specific effects [14]. Route and schedule of opioid administration may also influence $\mathrm{OIH}$.

An evidence-based review of available human studies concludes that although data is limited, the existence of $\mathrm{OIH}$ in humans is evident in normal volunteer studies utilizing brief opioid infusions. However, there is insufficient evidence to either support or refute the occurrence of OIH in the clinical setting [15]. Koppert et al. studied normal volunteers randomized to opioid infusion in a double-blind placebo-controlled design to assess hyperalgesia induced by electrical stimulation to the forearm [16]. Following induction with the conditioning stimulus hyperalgesia was quantified with von Frey filament mechanical stimulation. Subsequent measurements revealed attenuation of the hyperalgesic effect during a 30-min remifentanil infusion. However, post-infusion hyperalgesia was greater compared with non-opioid controls. Results indicate that following acute exposure to a potent opioid agonist subjects become more sensitive to noxious stimuli compared with opioid naïve controls. However, this study utilized a brief opioid infusion with resultant transient hyperalgesia and does not broadly apply to a clinically relevant scenario of chronic opioid therapy.

Although the quality of evidence with available studies in patients non-randomly exposed to opioid therapy is less robust, the outcomes are more illustrative of our evolving concept of OIH. One of the clearest experimental demonstrations of how OIH could manifest in humans utilizes the Cold Pressor Test. Doverty et al. compared the pain threshold and pain tolerance of 16 subjects on methadone maintenance therapy compared with 16 controls [17]. Subjects submerged one arm into an ice bath at time zero and were asked to endure as long as possible. Measured data points included onset pain (pain threshold) and time of voluntary withdrawal from the stimulus in seconds (pain tolerance). Figure 2 illustrates a significant variation between groups with respect to pain tolerance, although the threshold of pain perception was not statistically different. Many previously mentioned confounders such as non-randomization of opioid exposure, and possible pre-existing hyperalgesia in methadone maintenance subjects [18], preclude us from using this study to prove the existence of OIH in humans. Indeed, it would be a challenge to highlight a single study to either prove or refute the existence of a syndrome regardless of design quality. However, we may begin to understand how this entity could present clinically. 


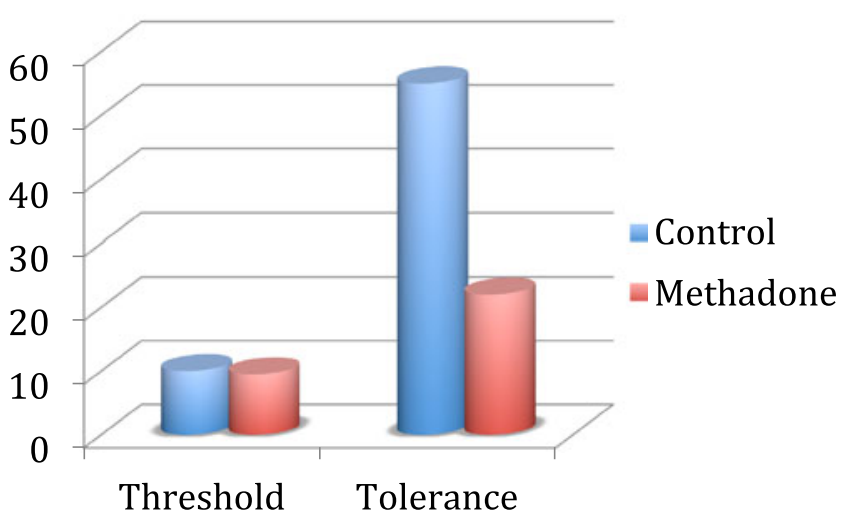

Fig. 2 Cold pressor test in methadone maintenance patients. This figure, adapted from Doverty et al. [17], compares the pain threshold and pain tolerance of controls with methadone maintenance subjects. Opioid withdrawal is controlled by conducting the experiment after daily dosing and by verifying methadone plasma concentrations. Placement of the forearm in an ice bath occurs at time zero. The $y$-axis measures seconds until subjects report pain (threshold) and again following self-termination of stimulus exposure (tolerance). Variation in pain threshold was not significant, whereas tolerance to the stimulus was markedly diminished in the methadone group

A deeper understanding of purported alteration in nociception derives from a study utilizing a research design known as Diffuse Noxious Inhibitory Control (DNIC). Ram et al. tested 110 chronic pain patients with respect to their rated pain intensity in response to a test stimulus [19]. Of these subjects, 73 were maintained on opioids chronically and 37 were treated for pain with non-opioid medications. All subjects were given an initial heat stimulus to the left hand and asked to report the pain intensity on a numeric pain scale (NPS). Subsequently, the right arm was submerged in an ice bath followed by application of second heat stimulus to the left hand. Subjects were asked to rate the second test stimulus using the NPS. Ordinarily, healthy volunteers would rate the second heat stimulus as less intense due to the effect of the conditioning ice bath that evokes anti-nociceptive pathways and modulates the perception of pain. The difference between the NPS rating of the first and second test stimulus, known as the magnitude of DNIC, measures the ability of intrinsic antinociceptive pathways to dampen pain signals. Comparing the magnitude of DNIC between groups in this study revealed significant impairment in anti-nociceptive pathways of subjects taking opioids chronically. The heterogeneity of enrolled subjects with respect to underlying pain conditions and prescribed opioid therapy introduces some limitations to data interpretation.

\section{Clinical Implications}

Many publications seek to further demonstrate $\mathrm{OIH}$ and define its characteristics with human experiments. Variations in measured outcomes, multiple modalities for induction of pain, inherent confounders, and lack of randomized exposure to opioids leave us without overwhelming proof that clinically significant $\mathrm{OIH}$ exists in humans. Regardless, animal data along with clinical observations suggest that OIH may contribute to inadequate pain control in patients maintained on long-term opioids. As a profession, we are reticent to undertreat pain, yet we are faced with the possibility that opioid treatment may actually worsen symptoms. Patients may expect a reflexive dose escalation in response to pain exacerbations, and could find suggestions of decreased opioid use or even termination of opioid therapy perplexing and unsatisfactory. Consideration of opioid rotation may present a valid and more palatable option to both patient and provider.

Although animal models of OIH are reproducible with a wide variety of opioids, rationale exists for rotating from one opioid to another when OIH is suspected. First, opioid rotation may avoid adverse effects associated with continued dose escalation of a single agent. Other considerations include interindividual variability in morphine receptor polymorphisms that affect binding affinities, and varied receptor subtype binding profiles among available opioid pharmaceuticals [20]. Current experience with treating presumed $\mathrm{OIH}$ in this manner remains anecdotal. Based on evidence from animal models demonstrating that NMDA receptor antagonists can prevent $\mathrm{OIH}$, agents such as methadone that not only bind opioid receptors, but also inhibit NMDA receptors, could provide a clever therapeutic substitution. However, long-term therapy with methadone appears to result in $\mathrm{OIH}$ as well, and this strategy may suffer limitations. Nevertheless, human studies producing OIH in response to brief infusions of remifentanil provide evidence that ketamine effectively limits hyperalgesia when coadministered, re-invigorating the concept of NMDA receptor antagonism as an effective maneuver [16]. One largescale human trial utilizing dextromethorphan, chosen for its NMDA receptor antagonism, in combination with morphine (MorphiDex ${ }^{\circledR}$ ) aimed to reduce pain scores and morphine requirements in chronic pain patients [21]. Though no significant effect was detected by the addition of dextromethorphan, the low doses administered may have limited efficacy.

While clinicians undoubtedly understand that opioid treatment suffers limitations, including incomplete pain control, neurocognitive and peripheral side effects, addiction, and drug diversion, the suggestion that opioids may actually worsen pain presents new challenges. Our current approach to management of chronic pain remains highly individualized with respect to opioid prescribing. Variables include length of therapy, agent of choice, degree and rapidity of dose escalation, assessment of efficacy, and grounds for termination of treatment. Suspicion of OIH may be limited to patients who self-report inadequate analgesia. Utilization 
of multiple prescribers in an effort to self-escalate dosing may obscure detection of treatment failures. Implementation of prescription monitoring programs in some states could aid physicians by providing additional feedback about their patients' opioid utilization. Enhancements in communication between primary care providers and other specialties such as emergency medicine remain paramount, though currently not well structured.

\section{Conclusions}

Animal models support clinical observations of OIH and lay the groundwork for developing a defined clinical description in humans. Current data provides an incomplete depiction of OIH and suffers from varied operational definitions of $\mathrm{OIH}$, lack of randomized exposure to opioids, and confounders associated with pre-existing disease states [22]. Controlled prospective randomized trials along with careful selection of research methods such as DNIC that are well suited to detect alterations in nociception are needed to reliably characterize the opioid-induced hyperalgesia syndrome. If reproducibly modeled, clinicians may utilize this data to establish broadly applicable clinical tools for assessment and treatment of patients maintained on long-term opioid therapy.

\section{References}

1. Von Korff M, Kolodny A, Deyo RA, Chou R (2011) Long-term opioid therapy reconsidered. Ann Intern Med 155(5):325-328

2. Nelson L, Goldfrank LR (2011) Goldfrank's toxicologic emergencies, 9th edn. McGraw-Hill Medical, New York

3. Ossipov MH, Lai J, King T, Vanderah TW, Malan TP Jr, Hruby VJ et al (2004) Antinociceptive and nociceptive actions of opioids. J Neurobiol 61(1):126-148

4. Vanderah TW, Suenaga NM, Ossipov MH, Malan TP Jr, Lai J, Porreca F (2001) Tonic descending facilitation from the rostral ventromedial medulla mediates opioid-induced abnormal pain and antinociceptive tolerance. J Neurosci 21(1):279-286

5. Collin E, Cesselin F (1991) Neurobiological mechanisms of opioid tolerance and dependence. Clin Neuropharmacol 14(6):465-488

6. Albutt C (1870) On the abuse of hypodermic injections of morphia. Practitioner 3:327-330

7. Lee M, Silverman SM, Hansen H, Patel VB, Manchikanti L (2011) A comprehensive review of opioid-induced hyperalgesia. Pain Physician 14(2):145-161

8. Hay JL, Kaboutari J, White JM, Salem A, Irvine R (2010) Model of methadone-induced hyperalgesia in rats and effect of memantine. Eur J Pharmacol 626(2-3):229-233
9. Angst MS, Clark JD (2006) Opioid-induced hyperalgesia: a qualitative systematic review. Anesthesiology 104(3):570-587

10. Avidor-Reiss T, Nevo I, Levy R, Pfeuffer T, Vogel Z (1996) Chronic opioid treatment induces adenylyl cyclase $\mathrm{V}$ superactivation. Involvement of Gbetagamma. J Biol Chem 271 (35):21309-21315

11. Mao J, Price DD, Mayer DJ (1994) Thermal hyperalgesia in association with the development of morphine tolerance in rats: roles of excitatory amino acid receptors and protein kinase C. J Neurosci 14(4):2301-2312

12. Arcaya JL, Cano G, Gomez G, Maixner W, Suarez-Roca H (1999) Dynorphin A increases substance $P$ release from trigeminal primary afferent C-fibers. Eur J Pharmacol 366(1):27-34

13. Belanger S, Ma W, Chabot JG, Quirion R (2002) Expression of calcitonin gene-related peptide, substance $\mathrm{P}$ and protein kinase $\mathrm{C}$ in cultured dorsal root ganglion neurons following chronic exposure to mu, delta and kappa opiates. Neuroscience 115(2):441-453

14. Lotsch J (2005) Opioid metabolites. J Pain Symptom Manag 29(5 Suppl):S10-S24

15. Fishbain DA, Cole B, Lewis JE, Gao J, Rosomoff RS (2009) Do opioids induce hyperalgesia in humans? An evidence-based structured review. Pain Med 10(5):829-839

16. Koppert W, Sittl R, Scheuber K, Alsheimer M, Schmelz M, Schuttler J (2003) Differential modulation of remifentanil-induced analgesia and postinfusion hyperalgesia by S-ketamine and clonidine in humans. Anesthesiology 99(1):152-159

17. Doverty M, White JM, Somogyi AA, Bochner F, Ali R, Ling W (2001) Hyperalgesic responses in methadone maintenance patients. Pain 90(1-2):91-96

18. Compton P, Canamar CP, Hillhouse M, Ling W (2012) Hyperalgesia in heroin dependent patients and the effects of opioid substitution therapy. J Pain 13(4):401-409

19. Ram KC, Eisenberg E, Haddad M, Pud D (2008) Oral opioid use alters DNIC but not cold pain perception in patients with chronic pain - new perspective of opioid-induced hyperalgesia. Pain 139 (2):431-438

20. Smith HS (2012) Opioids and neuropathic pain. Pain Physician 15 (3 Suppl):ES93-ES110

21. Galer BS, Lee D, Ma T, Nagle B, Schlagheck TG (2005) MorphiDex (morphine sulfate/dextromethorphan hydrobromide combination) in the treatment of chronic pain: three multicenter, randomized, double-blind, controlled clinical trials fail to demonstrate enhanced opioid analgesia or reduction in tolerance. Pain 115(3):284-295

22. Werner MU (2012) Vanguard research in opioid-induced hyperalgesia — but guard the basics. Pain 153(5):943-944

23. Vardanyan A, Wang R, Vanderah TW, Ossipov MH, Lai J, Porreca F et al (2009) TRPV1 receptor in expression of opioid-induced hyperalgesia. J Pain 10(3):243-252

24. Vanderah TW, Gardell LR, Burgess SE, Ibrahim M, Dogrul A, Zhong CM et al (2000) Dynorphin promotes abnormal pain and spinal opioid antinociceptive tolerance. J Neurosci 20 (18):7074-7079

25. Zhou Y, Sun YH, Zhang ZW, Han JS (1992) Accelerated expression of cholecystokinin gene in the brain of rats rendered tolerant to morphine. Neuroreport 3(12):1121-1123

26. Heinricher MM, McGaraughty S, Tortorici V (2001) Circuitry underlying antiopioid actions of cholecystokinin within the rostral ventromedial medulla. J Neurophysiol 85(1):280-286 